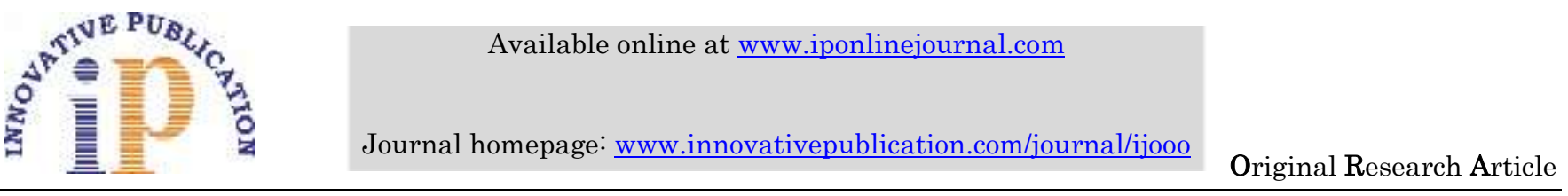

\title{
Treatment of chalazia by incision and curettage versus intralesional triamcinolone acetonide injection
}

\author{
Sirisha Brundavanam ${ }^{1}$, Ramya Deepthi ${ }^{2}$, Kumar Amruth ${ }^{3, *}$ \\ ${ }^{1}$ Junior resident, ${ }^{2,3}$ Associate Professor, ${ }^{1-3}$ Dept. of Ophthalmology, Narayana Medical College and Hospital, Nellore, Andhra Pradesh, \\ India
}

\begin{abstract}
Purpose: To compare the outcomes of treatment of primary chalazion with intralesional Triamcinolone Acetonide (TA) and Incision \& Curettage $(\mathrm{I} \& \mathrm{C})$ in patients with failed conservative treatment.

Materials and Methods

Study Design: Prospective randomized study

Place and Duration: The study was conducted from November 2017 to January 2019 in The Department of Ophthalmology, Narayana medical college, and Hospital, Nellore.

Methodology: 40 patients with primary chalazia who, after failed conservative treatment were randomized to either intralesional TA injection $(4 \mathrm{mg})$ or I\&C performed under local anesthesia. Preoperative photograph of the lesion was taken. Complete resolution was considered as lesion regression of $95 \%$ to $100 \%$. Treatment was considered a failure if no resolution was achieved after the first attempted I\&C or TA injection. Lesion resolution measured as $95 \%$ to $100 \%$ regression.

Results: Out of 40 patients, 20 were randomized to I\&C and 20 to TA injection group. Complete resolution was achieved in 16 cases in the I\&C group and 17 cases in TA group. The average time of resolution in TA group was four days. No significant complications were noted in either of the groups.

Conclusions: Intralesional TA injection is as effective as I\&C in cases of primary chalazia. So, injection may be considered as an alternative first-line treatment in cases where no biopsy is required.
\end{abstract}

Keywords: Chalazion, Incision \& curettage, Triamcinolone acetonide, Transconjunctival approach, Resolution.

\section{Introduction}

Chalazia are sterile lipogranulomatous inflammatory lesions that frequently become chronic and are usually caused by a blockage of meibomian gland ducts and the glands of Zeis, former being more common. They can be cosmetically disfiguring and, if large, may be associated with ptosis or visual disturbances due to acquired astigmatism. Spontaneous regression can occur in $25 \%$ to $43 \%$ lesions ${ }^{1}$. Patients are initially advised to apply hot compresses $(\mathrm{H} / \mathrm{C})$ to the cyst to facilitate spontaneous drainage along with lid hygiene and digital massage. In case this fails, other treatment options are incision and curettage (I\&C) under a local anesthetic injection, intralesional steroid injections (SI), a combination of intralesional SI with I\&C, and total excision.

Conservative therapy is the least invasive, but it requires persistent effort from the patient because there may be a need for treatment up to 4 times a day for a few weeks. The success rate of conservative therapy is greatly dependent on patient education by the physician and patient compliance with the treatment regimen. ${ }^{1-4}$

Incision and curettage involves topical and infiltrated anesthetic, eversion of the eyelid with a clamp, vertical incision, removal of the capsule, and its contents by curettage, and patching the eye. Topical or systemic antibiotics may be required after the procedure for 5-7 days. ${ }^{5}$

Intralesional Triamcinolone acetonide (TA) injection of volume 0.02 to $1 \mathrm{ml}$ in the concentration of 5 to $40 \mathrm{mg} / \mathrm{ml}$ in transcutaneous or transconjunctival routes is being considered as an alternative to incision and curettage (I\&C). Although transcutaneous injection is less painful for the patient, the transconjunctival route is preferred due to reports of incidence of depigmentation at the injection site, globe perforation and anterior segment ischemia in transcutaneous route. ${ }^{6,7}$ Retinal vascular occlusion is a complication, probably due to intravascular injection with subsequent embolization. However, for marginal lesions and lesions closer to structures like lacrimal punctum, this method is preferred. ${ }^{5}$ With this background, we take up this study to focus on comparing the results of Incision and Curettage (I\&C) and Intralesional TA injection for the treatment of primary chalazia.

\section{Materials and Methods \\ Study Design \\ Prospective randomized study}

\section{Study Period}

November 2017 to January 2019.

\footnotetext{
*Corresponding Author: Kumar Amruth, Dept. of Ophthalmology, Narayana Medical College and Hospital, Nellore, Andhra Pradesh, India

Email: amruth.chavan@gmail.com

http://doi.org/10.18231/j.ijooo.2019.034
} 


\section{Study Subjects}

Patients with primary chalazia are selected for surgical intervention from the Out Patient Department (OPD) at the Department of Ophthalmology.

Sample Size: 40 cases with primary chalazia

\section{Ethical clearance and conflict of interests}

The study proposal was cleared by the Institutional Research Board before the beginning of data collection. There was no conflict of interests involved.

There were no external funding sources. No financial interests involved.

\section{Inclusion criteria}

1. Patients above 18 years

2. Palpable chalazion and failure of conservative treatment

3. Normal anatomy of the lid

\section{Exclusion criteria}

1. Patients below 18 years

2. Recurrent chalazia

3. Multiple chalazia on the same lid

4. Abnormal lid tissue/ lashes

5. Presence of associated eyelid infection

6. Patients allergic to the drugs used

7. Suspicion of malignancy

8. Patients unable to/ refuse to give consent

\section{Methodology}

Informed consent had been taken from all the patients who met the inclusion criteria and were willing to take part in the study. They failed to respond to conservative therapy for chalazia, including hot compresses, lid hygiene, and local antibiotic ointment. Incision and curettage (I\&C) and Triamcinolone Acetonide (TA) injection procedures were done by a single surgeon.

The following points were noted: name, age, sex, address, occupation, history, general examination, local examination. All of them underwent detailed ophthalmic examination, including Visual Acuity (VA), intraocular pressure (IOP), oblique examination, slit-lamp examination, and slit-lamp biomicroscopy.

The otherwise normal patients except for chalazion were randomly divided into two groups for chalazion treatment by either Incision and Curettage (I\&C) or Triamcinolone Acetonide (TA) injection. Size of the lesion was noted for the first time of presentation and at each postoperative visit. They were followed up at 1 week, 2 weeks, and 6 weeks. Complete resolution was taken as lesion regression of $>90 \%$. Complete resolution was noted in 18 cases in the I\&C group and 16 cases in the TA group. Most of the patients had complete resolution.

Technique of TriamcinoloneAcetonide injection (TA): Topical anesthesia eye drops were instilled, the area surrounding the lesion was cleaned with $5 \%$ povidoneiodine, and the eyelid was everted, and a 30 gauge needle on
$1 \mathrm{~mL}$ insulin syringe was used to inject $0.1 \mathrm{ml}$ of TA $40 \mathrm{mg} / \mathrm{ml}$ intralesionally in transconjunctival route. Massage was done over the injected site. No eye patching was done.

Technique of Incision \& Curettage (I\&C): The eyelid was infiltrated with 2 to $3 \mathrm{~mL}$ of lidocaine $2 \%$ with 1:10,000 Adrenaline using a 25Guaze needle. Topical Betadine solution 5\% was used to prepare the eyelid, and the procedure was performed under sterile conditions in the minor Operation Theatre room. The eyelid was everted, and a chalazion clamp was placed. A single vertical incision was made at the point of the lesion, and all pus material was cleaned using a curette, breaking the loculations and the lesion's capsule was incised and removed. Chloramphenicol with Hydrocortisoneacetate ointment was applied, and the eye was bandaged for 6 hours. The ointment is to be applied four times a day for five days.

\section{Statistical analysis}

The data was summarized in Microsoft Excel. To compare the results of two groups (complete resolution of chalazion among I\&C group versus TA group) Chi-square test was used. Lack of response to either method after the first attempt was considered treatment failure.

\section{Results}

Forty patients were included in the study. Demographics were tabulated as below:

Table 1: Demographic data of the two groups

\begin{tabular}{|c|c|c|}
\hline & $\begin{array}{c}\text { Incision \& } \\
\text { Curettage (I\&C) }\end{array}$ & $\begin{array}{c}\text { triamcinolone acetonide } \\
\text { injection (TA) }\end{array}$ \\
\hline Number & 20 & 20 \\
\hline Age & $30+/-12$ & $32+/-11$ \\
\hline Gender & & 9 \\
Male & 12 & 11 \\
Female & 8 & 9 \\
\hline
\end{tabular}

Of the 20 patients in I\&C group, seven had chalazion over the right upper lid; four had it over right lower lid, five over the left upper lid and four over left lower lid. Among 20 patients in the group, eight showed chalazion over the right upper lid, three over right lower lid, six over the left upper lid and three over left lower lid.

Patients were followed up for six weeks. Complete resolution was observed in 18 patients $(90 \%)$ in the I\&C group and 16 patients $(80 \%)$ in the TA group. There is no significant difference in the rates of resolution between the two groups $(p>0.05)$. Complete lesion regression was considered if $>90 \%$ size reduction was observed. Average time of resolution was 11 days in the TA group. The four patients that failed to respond to TA were repeated with TA injection, and three of them showed resolution, while one needed I\&C again. Of the two patients who had no complete resolution with $I \& C$ were subjected to repeat I\&C. No recurrence was noted in either of the groups. No significant complications were encountered in either of the procedures. 


\section{Discussion}

Our results showed that intralesional TA injection is as effective as I\&C for the successful treatment of primary chalazia. Most lesions resolved with one injection, and only a few required two injections. TA injection is a simpler and less inconvenient method, though a bit painful to the patient. Goawalaa and associates compared intralesional TA $2 \mathrm{mg}$ with I\&C and warm compresses and found similar resolution rates for TA and I\&C $(84 \%$ and $87 \%$, respectively) to be significantly higher than only conservative treatment $(46 \%)^{7}$ This is in support of our study. Steroid injection into the lesion was reported to give similar results as I\&C. It can be preferred for marginal lesions or lesions close to structures such as lacrimal punctum due to the risk of surgical damage ${ }^{5}$. Khurana and associates stated that intralesional therapy is equally effective in small and marginal chalazia, whereas larger suppurating lesions may respond better to surgery (I\&C) than to injections. ${ }^{8}$ The advantages of $\mathrm{I} \& \mathrm{C}$ include that it can be used in infected chalazia and those which necessitate biopsy, such as recurrent chalazia. ${ }^{9}$ I\&C is also recommended for treatment of larger lesions. ${ }^{8}$ However, $\mathrm{I} \& \mathrm{C}$ requires more equipment and expertise, has a longer recovery time, and has some adverse events including delayed bleeding, lacrimal punctual scarring, and loss of functionality. ${ }^{9,10}$ Mohan $\mathrm{K}$ and associates reported good response to steroid injections in up to $92 \%$ of the cases, regardless of the duration or consistency of the chalazion ${ }^{11}$.

The most common adverse effect of intralesional steroid injection is local skin depigmentation, encountered with the transcutaneous approach. The transconjunctival approach minimizes this, and it also minimizes the risk of inadvertent penetration of the globe. $\mathrm{Ho}^{12}$ documented that two out of 48 patients who underwent intralesional triamcinolone acetonide injection were affected by localized skin depigmentation. The reason could be that although the injection is aimed at being deposited intralesionally, a small portion of triamcinolone acetonide (TA) may be deposited by the needle on the way in or out of the injection site. In the Goawalla study, none of the 56 patients that underwent this procedure suffered this adverse effect. ${ }^{7}$

None of our subjects had complications from the TA injection, but clinicians carrying out such procedure should, however, be aware of the potential complications including yellowish deposits at the injection site, elevated IOP, and skin hypopigmentations, globe perforation, microembolization, and retinal/choroidal vascular occlusions.

The average time of resolution of the chalazion after one triamcinolone acetonide (TA) injection in our study was 11 days, which is in correlation with the study of Simon et al. ${ }^{13}$ In studies by Biuk et al. mean resolution time of 2.5 weeks was noted. ${ }^{14}$ In studies by Mustafa and Oriafage majority showed resolution in 2-4 weeks, ${ }^{9}$ and in studies by Watson and Austin, the average resolution time is within three weeks. $^{15}$

\section{Conclusion}

Ours and several previous reports support intralesional steroid injections for the treatment of eyelid chalazia. TA injection can be offered to all patients when a biopsy is not required. If treatment by 1 or 2 injections fails, then I\&C should be recommended. TA injections may be more beneficial in marginal chalazia or in those located near the lacrimal puncta by avoiding excess scarring with the resultant cosmetic or functional disturbances.

\section{Source of Funding}

None.

\section{Conflict of Interest}

None.

\section{References}

1. Cottrell DG, Bosanquet RC, Fawcett IM. chalazion: the frequency of spontaneous resolution. Br Med J 1983;287:1595.

2. Bohigian GM. Chalazion: a clinical evaluation. Ann Ophthalmol 1979;11:1397-8.

3. Garrett GW, Gillespie ME, Mannix BC. Adrenocorticosteroid injection vs. conservative therapy in the treatment of chalazia. Ann Ophthalmol 1988;20:196-8.

4. Perry HD, Serniuk RA. Conservative treatment of chalazia. Ophthalmol 1980;87:218-21.

5. Kanski Jack J, Brad Bowling. Kanski's Clinical Ophthalmology. Eighth. 2016.

6. Ahmad S, Baig MA, Khan MA. Intralesional corticosteroid injection vs surgical treatment of chalazia in pigmented patients. J Coll Physicians Surg Pak 2006;16:42-4.

7. Goawalla A, Lee V. A prospective randomized treatment study comparing three treatment options for chalazia: triamcinolone acetonide injections, incision and curettage and treatment with hot compresses. Clin Experiment Ophthalmol 2007;35:706-12.

8. Khurana AK, Ahluwalia BK, Rajan C. Chalazion therapy: Intralesional steroids versus incision and curettage. Acta Ophthalmol (Copenh) 1988;66(3):352-4.

9. Mustafa TA, Oriafage IH. Three methods of treatment of chalazia in children. Saudi Med J 2001;22:968-72.

10. Prasad S, Gupta AK. Subconjunctival total excision in the treatment of chronic chalazia. Indian J Ophthalmol 1992;40:103-5.

11. Mohan K, Dhir SP, Munjal VP, Jain IS. Use of intralesional steroids in the treatment of chalazion. Ann Ophthalmol 1986;18(4):158-60.

12. Hosy, Lai JSM, HKMJ 2002;8:18.

13. Ben simon GJ, Huang L, Nakra T, Schwarcz RM, McCann JD, Goldberg RA. Ophthalmol 2005;112:913.

14. Biuk D, Matić S, Barać J. Chalazion management. Surgical treatment versus triamcinolon application. Coll Antropol 2013;37:247-50.

15. Watson AP, Austin DJ. Treatment of chalazions with injection of a steroid suspension. Br J Ophthalmol 1984;68:833-5.

How to cite this article: Brundavanam S, Deepthi R, Kumar A. Treatment of chalazia by incision and curettage versus intralesional triamcinolone acetonide injection, Int $J$ Ocul Oncol Oculoplasty 2019;5(3):133-5. 